\title{
Clusterin in Stool: A New Biomarker for Colon Cancer Screening?
}

Sabina Pucci, $\mathrm{PhD}^{1}$, Elena Bonanno, $\mathrm{MD}^{1}$, Fabiola Sesti, $\mathrm{PhD}^{1}$, Paola Mazzarelli, $\mathrm{MD}^{1}$, Alessandro Mauriello, $\mathrm{MD}^{1}$, Federico Ricci, $\mathrm{MD}^{2}$, Giuseppe Biondi Zoccai, MD³, Francesco Rulli, $\mathrm{MD}^{4}$, Gabriele Galatà, MD ${ }^{4}$ and Luigi G. Spagnoli, MD ${ }^{1,5}$

OBJECTIVES: The identification of useful markers for early diagnosis of human colon cancer is a major goal still in progress. Clusterin is a pleiotropic protein with a broad range of functions. It has recently drawn much attention because of its association with cancer promotion and metastasis. It is involved in prosurvival and apoptosis processes that are carried out by two different isoforms. Secreted clusterin isoform (sCLU) is cytoprotective and its prosurvival function is the basis of the current phase I/II clinical trials against prostate, lung, and breast cancers. We have already shown that in colorectal cancer (CRC) there is an increased expression of sCLU. In this report, we investigated whether sCLU is released in the blood and stool of colon cancer patients in order to study SCLU as a potential diagnostic molecular marker for colon cancer screening.

METHODS: $\quad$ The quantitative expression of SCLU was determined by dot blot immunodosage in the serum and stool of CRC patients $(n=63)$ and age-matched controls without clinical history of neoplasia, CRC, or systemic or bowel inflammatory disease $(n=50)$. Unpaired $t$-tests and Mann-Whitney $U$-tests were used for continuous variables. The diagnostic performance of clusterin was appraised by means of receiver operating characteristic (ROC) curves.

RESULTS: $\quad$ We found a significant increase of $s C L U$ in the serum and stool of CRC patients $(P=0.0002$ and $P<0.000$, respectively) as compared with controls. ROC curves provided cutoff points showing a trade-off between sensitivity and specificity. With a cutoff point of $88.5 \mu \mathrm{g} / \mathrm{ml}$, sCLU in blood showed a $55.6 \%$ sensitivity and $100 \%$ specificity, and with a cutoff point of $34.6 \mu \mathrm{g} / \mathrm{g}$, the stool test reached $66.7 \%$ sensitivity and $84 \%$ specificity in discriminating between nonneoplastic and colorectal neoplastic lesions. Human cancer xenografts in nude mice indicated a positive correlation between increasing serum clusterin level and tumor size.

CONCLUSIONS: This study highlights the potential of clusterin detection in stool to be a valuable tool to improve the effectiveness and efficiency of large-scale clinical cancer screening.

Am J Gastroenterol advance online publication, 21 July 2009; doi:10.1038/ajg.2009.412

\section{INTRODUCTION}

Colon cancer is currently the third most common cancer diagnosed among men and women and the second most common cause of death after lung cancer in the Western countries. A higher incidence of colon cancer is observed between the ages of 60 and 70 , and $60 \%$ of the patients survive up to 5 years (1). The most important reason for the low percentage of recoveries is the fact that when the primary tumor is removed, a high number of patients have already developed micro-metastases, principally at the liver. Therefore, methods for early screening are requested. At present, the early diagnosis protocols (secondary prevention) consist of rectal exploration, determination of fecal occult blood test and rectosigmoidoscopy periodically performed on individuals of 45 years of age, and on older and nonsymptomatic individuals. Periodic pan-colonoscopy is the only procedure for early diagnosis of

\footnotetext{
'Department of Biopathology, University of Rome "Tor Vergata," Rome, Italy; ${ }^{2}$ Department of Biopathology, Section of Ophthalmology, University of Rome "Tor Vergata," Rome, Italy; ${ }^{3}$ Interventional Cardiology, Division of Cardiology, University of Turin, S. Giovanni Battista "Molinette" Hospital, Turin, Italy; ${ }^{4}$ Department of Surgery, University of Rome "Tor Vergata," Rome, Italy; ${ }^{5}$ RCCS, San Raffaele, Laasana, Rome, Italy. Correspondence: Sabina Pucci, PhD, or Luigi G. Spagnoli, MD, Department of Biopathology, University of Rome "Tor Vergata," Via Montpellier 1, Rome 133, Italy. E-mail: sabina.pucci@uniroma2.it or spagnoli@uniroma2.it Received 28 January 2009; accepted 11 June 2009
} 
neoplasia in individuals with positive familial history of colorectal cancer (CRC), in patients already having a neoplasia or affected by a syndrome with a high risk of neoplasia insurgence, who are part of the so-called "at-risk population" (2). Randomized controlled trials have shown that annual or biennal screening in asymptomatic people over the age of 50 years using fecal occult blood tests can reduce CRC mortality by 15-33\%. Nevertheless, fecal occult blood test, used for early diagnosis of colon carcinoma in clinical practice, yields frequent false-negative and false-positive results that lower the screening effectiveness and raise the program costs $(3,4)$. On the basis of the above, new molecular pathogenetic markers that would overcome the restrictions of the invasive methods used at present, such as colonoscopy, are needed to improve the efficacy, sensitivity, and specificity of early diagnosis tests $(5,6)$. Moreover, molecular markers specific for this pathology would help to stratify more selectively the cohort of patients really needing colonoscopy.

One of the signatures of a cancer cell is change in the nuclear and cytoplasmic structure and architecture. In particular, molecular changes in cell cycle or apoptosis-related protein expression and localization are associated with different types of cancers. Recent studies have shown the increased expression of clusterin in cancer, suggesting a role of this protein in tumor progression $(7,8)$.

Clusterin is a heterodimeric ( $\alpha$ - and $\beta$-chains) ubiquitous glycoprotein implicated in a large number of physiological processes and in the control of cellular proliferation. A number of isoforms have been characterized that differ in the grade of glycosylation and in their function $(9,10)$.

Several experimental data have shown a strong correlation between the presence of the different isoforms of clusterin and tumoral progression (7). The disappearance of the proapoptotic nuclear form (nCLU, $60 \mathrm{kDa}$ ) and the overexpression of the prosurvival secreted/cytoplasmatic isoform (sCLU, $40 \mathrm{kDa}$ ) marks the transition from normal cell to neoplastic phenotype. Besides, with the acquisition of aggressiveness, the disappearance of the proapoptotic CLU strongly associates with the loss of DNA repair activity of the complex Ku70/80 (11) and with the overexpression of the prosurvival-secreted form (sCLU). The expression of sCLU isoform directly correlates with tumor aggressiveness and with the metastatic potential of the tumor. It seems reasonable that the shift of CLU production can be related to exogenous factors such as interleukin- 6 and transforming growth factor- $\beta$ that are present in the extracellular milieu of the tumoral mass (12). Moreover, the increase of sCLU in tumors suggests that this isoform could be released in the extracellular space.

The results concerning the modulation of different CLUs (proapoptotic nCLU and prosurvival sCLU) in the tumorigenesis of CRC suggest the possible role of sCLU as a potential new marker for the diagnosis and prognosis of CRC.

The use of clusterin as a diagnostic marker in some pathological conditions such as type II diabetes and several coronary pathologies has already been described (13). There were just a few earlier attempts to determine clusterin by enzyme-linked immunosorbent assay (ELISA) in tumoral pathologies, specifically in the blood of prostate carcinoma patients (14). Moreover, clusterin level in the blood and urine has been shown to be a potential marker for bladder and kidney tumors, being directly related to the dimension of the neoplasia (15).

In this report, we have highlighted that the appearance and progressive increase of the clusterin cytoplasmic isoform in tumors correlate with a significant increase of clusterin in the serum and stool of patients affected by CRC. Furthermore, we confirmed the positive correlation between the increasing serum clusterin level and tumor size, in human cancer xenograft in nude mice.

On the basis of these observations, we have currently also validated a new method for blood and stool immuno-dosage of clusterin in colon cancer patients by the use of specific oligoclonal antibodies, recognizing selectively the secreted isoform of clusterin.

\section{METHODS}

\section{Case selection}

Consecutive patients with suspected colon cancer undergoing gastrointestinal endoscopy, willing to participate in the study and providing written informed consent, were enrolled at the departments of general surgery of our institution. This being a feasibility and pilot study, the first ever to test the diagnostic role of clusterin in patients with colon cancer, no formal sample size computation was performed, and the study sample was mainly chosen given the typical availability of pathologic specimens during the study period ( 1 year). The participants enrolled in the study are reported in Table $\mathbf{1}$. None had preoperative chemotherapy or irradiation. The clinical and histopathogical features of the tumors selected for blood and stool studies are reported in Table 2. Age-matched controls were enrolled at the Internal Medicine Clinics of our Institution, after confirmation of lack of clinical history of neoplasia, CRC, systemic or bowel inflammatory disease, and no diagnosis of diabetes or autoimmune pathologies. This study was approved by the local Ethics Committee.

\section{Sample collection}

Blood samples were obtained before surgery from patients with $\mathrm{CRC}$ and from no-CRC age-matched patients (controls), to determine the serum concentration of clusterin. After clotting, the blood samples were centrifuged at 2500 r.p.m. for $15 \mathrm{~min}$ and the serum was removed, aliquoted, and stored at $-80^{\circ} \mathrm{C}$ until analysis.

Stool samples were obtained before surgery from patients with $\mathrm{CRC}$ and from no-CRC age-matched subjects (controls). Moreover, feces samples $(n=10)$ were collected from autoptic examination (performed within $6 \mathrm{~h}$ from death), resulting in negative for any neoplasia and gastrointestinal diseases at the macroscopic evaluation of whole gastrointestinal apparatus, and were used as controls (Table 1). Stools were homogenized, and about $100 \mathrm{mg}$ of each sample were suspended in stool extraction buffer by ScheBo Biotech AG (Giessen, Germany) at a concentration 


\begin{tabular}{|c|c|c|c|c|c|}
\hline & $n$ & Males & Females & Median age (years) & Range (years) \\
\hline CRC patients & 35 & 23 & 12 & 65.4 & $39-82$ \\
\hline Controls & 25 & 10 & 15 & 63.5 & $36-85$ \\
\hline CRC patients & 28 & 16 & 12 & 65.5 & $43-80$ \\
\hline Controls $^{a}$ & 29 & 12 & 17 & 64.1 & $34-82$ \\
\hline
\end{tabular}

\section{Table 2. Blood and stool study: clinico-pathological characteristics of CRC patients}

\begin{tabular}{|c|c|c|}
\hline Factor & Blood $(n=35)$ & Stool $(n=28)$ \\
\hline \multicolumn{3}{|l|}{ Location of tumor } \\
\hline Colon right & 8 & 9 \\
\hline Colon left & 25 & 17 \\
\hline Rectum & 2 & 2 \\
\hline \multicolumn{3}{|l|}{ Histology } \\
\hline Well to moderately differentiated & 29 & 23 \\
\hline Poorly differentiated & 6 & 5 \\
\hline \multicolumn{3}{|l|}{ TNM stage } \\
\hline $\mathrm{T} 1$ & 3 & 2 \\
\hline $\mathrm{T} 2$ & 12 & 6 \\
\hline T3 & 15 & 20 \\
\hline $\mathrm{T} 4$ & 5 & 0 \\
\hline \multicolumn{3}{|l|}{ Lymph node metastasis } \\
\hline NO & 28 & 22 \\
\hline $\mathrm{N} 1-2$ & 7 & 6 \\
\hline \multicolumn{3}{|l|}{ Duke's stage } \\
\hline A & 0 & 0 \\
\hline B & 25 & 21 \\
\hline C & 8 & 5 \\
\hline $\mathrm{D}$ & 2 & 2 \\
\hline
\end{tabular}

of $10 \mathrm{mg} / \mathrm{ml}$. The protein extract was diluted 1:20 in phosphatebuffered saline, and stored at $-80^{\circ} \mathrm{C}$ until analysis.

\section{Morphological and Immunohistochemical studies}

Hematoxylin and eosin sections were evaluated for the diagnosis and clinical staging of CRC (assessed according to the Dukes' and TNM classifications).
After a selection of the most significant area of the neoplasia, a tissue microarray (TMA) was constructed including the cancer and normal mucosa of 50 CRC patients (28 tumors from the patients enrolled in the stool study, and 22 tumors from the archival paraffin blocks). Tissue cores $(1.2 \mathrm{~mm}$ diameter) were arrayed into the "host" paraffin block using TMA CK3500 (BioRep, Milan, Italy). The TMA slides were incubated with the rabbit polyclonal antibody clusterin- $\alpha$ (H-330) or the goat polyclonal antibody clusterin- $\beta$ (M-18) (Santa Cruz Biotechnology, Santa Cruz, CA). Specimens were also immunostained with the anti-Clu-C2 antibody produced by us (patent RM2004A000098), directed against the sCLU. The evaluation of the TMA immunohistochemistry staining was confirmed by three independent observers unaware of the clinical data and molecular results (7). The concordance between TMA and full sections was tested for all the antibodies in 10 specimens selected randomly.

Tissue staining was semiquantitatively graded for intensity from negative: 0 to strong $(3+++)$. Clusterin protein expression was deemed positive when weak $(+/-)$ to strong $(+++)$ staining was present (12).

\section{Ex vivo cell culture and animal experimental model}

Tumoral tissue and normal mucosa from the same patient were selected on hematoxylin and eosin frozen sections. Primary cell cultures were performed: $10 \times 10^{7}$ neoplastic and nonneoplastic colonic cells, ex vivo isolated, from tumoral and healthy colonic mucosa, were separately incubated in complete culture medium for $72 \mathrm{~h}$ at $37^{\circ} \mathrm{C}$ in a $5 \% \mathrm{CO}_{2}$ humidified incubator. Supernatants were stored at $4^{\circ} \mathrm{C}$.

Human colon carcinoma (HTB-37, ATCC) Caco-2 cells were grown according to the condition suggested from ATCC. Caco-2 cells were subcutaneously injected in the right flank of six nude mice $\left(10 \times 10^{6} \mathrm{Caco}-2\right.$ cells/mouse). Before tumor cell injection, $500 \mu \mathrm{l}$ of blood were collected from each animal to determine the basal serum concentration of clusterin in mice. Mice were killed at days 15, 20, and 25 from tumor injection. At the killing of each mouse, blood was collected as described above and the tumor size determined. 


\section{Enzyme-linked immunosorbent assay}

A sandwich ELISA was performed as described earlier (13), with the following modifications: the antibodies used were a mouse monoclonal antibody (anti-clusterin- $\alpha$ chain, clone $41 \mathrm{D}$, Upstate, NY) to coat the plates, a rabbit oligoclonal anti-sCLU antibody (C3) that we have produced (patent RM2004A000098), and a rabbit horseradish peroxidase-linked whole antibody (Amersham, GE Healthcare, $\mathrm{UK}$ ) as primary and secondary antibodies. The $\mathrm{C} 3$ oligoclonal antibody was obtained by the selection of short antigenic epitopes specific for the sCLU isoform. Serum samples were diluted 1:8. Standard curves were generated with human recombinant clusterin (Alexis Biochemicals, San Diego, CA). A competitive ELISA kit (AdipoGen, Korea) for human clusterin was also used. Plates were read in an absorbance reader (Sunrise-Basic, Tecan, Switzerland).

\section{Western blot and dot blot analysis}

For western blot, $40 \mu \mathrm{l}$ of the stool extract of each sample was loaded on $12 \%$ SDS-denaturing polyacrylamide gels. Dot blot analysis was performed using $3 \mu \mathrm{l}$ of serum or stool extracts denaturated in sample, and each sample was loaded on a polyvinyl-diene difluoride membrane membrane.

In both cases, the detection of clusterin was performed by the rabbit oligoclonal antibody anti-Clu-C2 produced by us (patent RM2004A000098). Standard curves were generated with human recombinant clusterin (Alexis Biochemicals).

\section{Statistical analysis}

All values provided in the text and figures are the mean of three independent experiments \pm s.d. For immunohistochemical results, the statistical significance was calculated by comparing the intensity values (from 0 to 3 ) between different groups, e.g., CLU staining values in normal mucosae vs. adenocarcinomas (CRC). Unpaired $t$-tests were used for normally distributed continuous variables and Mann-Whitney $U$-tests were used for continuous variables not following a normal distribution (as shown by significant results after Kolmogodorov-Smirnov tests). Calculations and upper and lower bounds for confidence intervals (CI) were performed by nonparametric assay and SPSS software (SPSS, Chicago, IL) (two tailed; $P \leq 0.05$ was significant).

The diagnostic performance of clusterin was appraised by means of receiver operating characteristic (ROC) curves, with corresponding area under the curve (AUC) (16). In addition, the AUCs of ROCs from the stool and blood clusterin assay were compared according to Vergara et al. (17) Every case or control sample was measured three times for clusterin levels, and the averaged value was used for ROC curves. The ROC curves provided several cutoff points to show the trade-off between sensitivity and specificity at different cutoff values. Sensitivity and specificity values were calculated with confidence interval analysis according to the Wilson method.

\section{RESULTS}

\section{sClusterin is overexpressed in colon cancer}

Expression of CLU (nCLU and sCLU isoforms) was analyzed by immunohistochemistry in human colon cancer tissues $(n=50)$ and in normal mucosa aside from the neoplasia $(n=50)$. Participants enrolled in this study, and their clinical and histopathological features are reported in Table $\mathbf{1}$ and Table 2. CLU immunostaining was performed with anti-CLU C2 that we produced and confirmed by the use of commercial antibodies as described earlier $(7,12)$. CLU was expressed quite exclusively in the nuclei of normal mucosa, whereas nuclear CLU staining was not detected in all carcinomas observed. On the other hand, cytoplasmic CLU expression, faintly present in normal mucosa, was strongly expressed in all colon carcinomas (normal mucosae vs. carcinomas: $P=0.02$ ) (Figure 1) and its upregulation correlated with the node status (any T, N0 vs. any T, N1: $P=0.04$ ). To define whether the overexpression of sCLU in the cytoplasm of tumors was correlated with an increased extracellular release, tumoral cells and normal mucosa were aseptically isolated from surgical biopsies and incubated for $72 \mathrm{~h}$. Culture supernatant from the ex vivo-isolated cells of healthy and neoplastic colonic mucosa was collected and the clusterin level was evaluated by ELISA. A significant increase of the clusterin level (2.9 times, $P=0.03$ ) was found in the culture supernatant of tumoral cells, compared with healthy mucosa cells of the same patient (Figure 2a).

\section{Serum clusterin level correlates with tumor size in a tumor xenograft model}

To investigate whether clusterin release from colon cancer cells could effectively affect the total amount of circulating protein in blood, $10 \times 10^{6}$ human colon cancer cells, Caco-2, were underskin injected in nude mice $(n=6)$ after blood was drawn for CLU endogenous basal level determination. To evaluate the CLU level in relation to tumor size, mice were killed at days $15(n=2), 20(n=2)$, and $25(n=2)$ after tumor injection. As shown in Figure 2b, the level of clusterin, evaluated by dot blot analysis, was significantly increased in the blood of tumor-bearing mice, compared with uninjected mice. Data showed that tumor cells are able to release clusterin and that the increasing level of clusterin in the blood of tumor-bearing mice as compared with tumor-free mice, evaluated in different times of tumor growth, is exclusively related to tumor presence. Moreover, the rise of clusterin serum levels was related to the tumor volume increase (Figure $\mathbf{2 b}$ ).

\section{Clusterin level in blood increases in colon cancer patients}

The quantitative determination of sCLU in biological fluids (blood and stool) was developed by adapting the ELISA technique described earlier (13). A sandwich ELISA was performed using a rabbit oligoclonal antibody raised against the secreted glycosylated isoform of human clusterin, anti-CLU C3, that we have produced and with a commercial antibody. The results obtained were confirmed by commercial ELISA, as described in the Methods section. As shown in Figure 3a, clusterin levels did not significantly differ in sera between cancer patients and healthy individuals $(P=0.07$ Student's $t$-test). Moreover, the reproducibility of the results was strongly influenced by the clustering properties of this stress-folding 

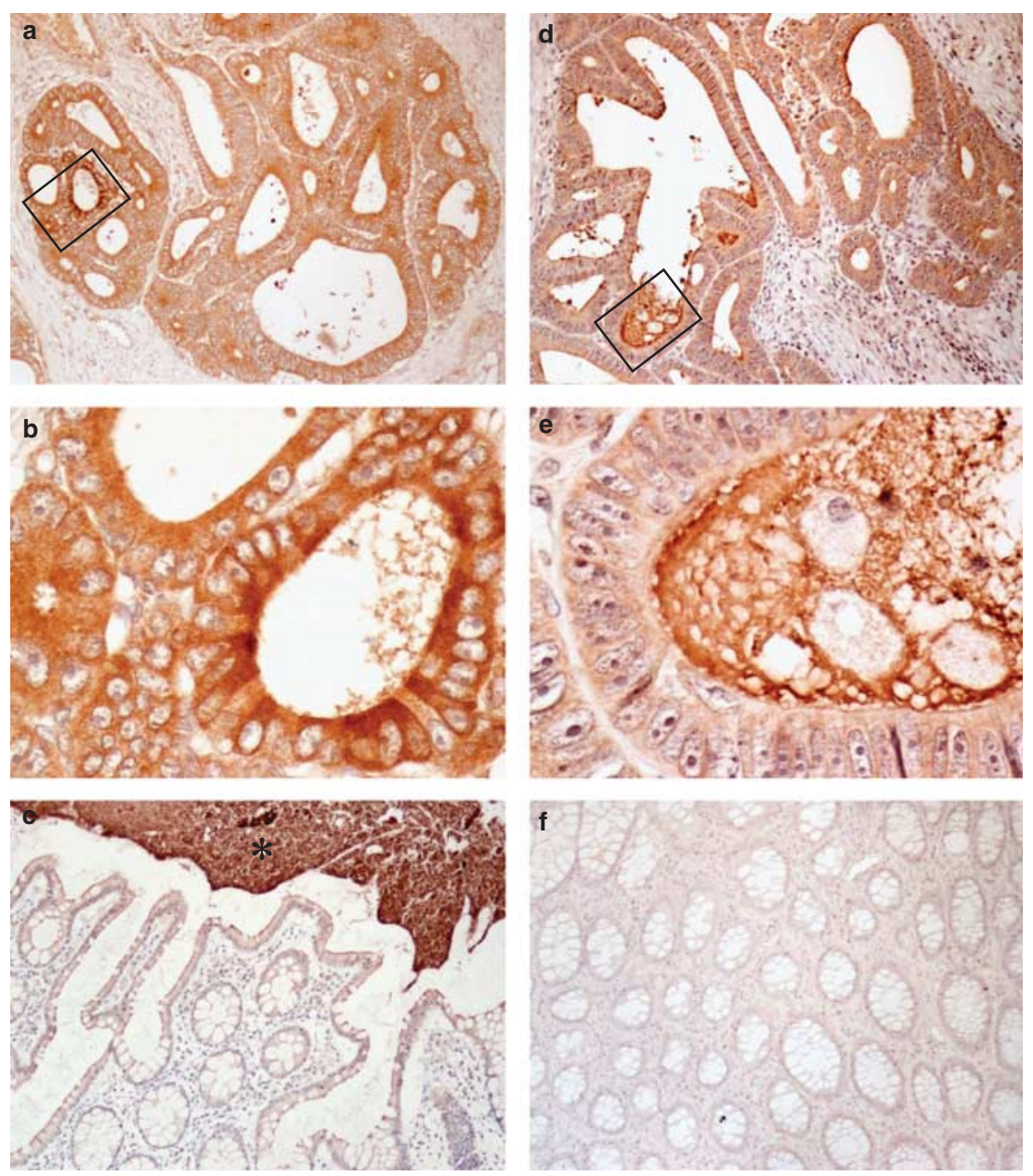

Figure 1. Immunohistochemical analysis of clusterin expression in colorectal cancer (CRC) patients. A low-power field (magnification $\times 10$ ) of a G2, pT3N1 CRC patient (a). A diffuse and intense positive reaction for the anti-Clu-C2 antibody (specific for the secreted form of clusterin isoform (CLU)) was observed. (b): Higher magnification (x60) of the insert in panel a. The cytoplasm of glandular cells displayed a strong positive reaction, whereas the nuclei were completely negative. (c) A low-power field (magnification $\times 10$ ) of normal mucosa of the same patient aside from the neoplasia. The epithelial lining at the luminal front of the colon was weakly positive for the anti-CLU antibody; on the other hand, the fecal content observed in the lumen was diffusely positive. (d) Low-power field (magnification $\times 10$ ) of a G2, pT2NO CRC patient showed a diffuse positive reaction for the anti Clu-C2 antibody. (e) Higher magnification ( $\times 60$ ) of the insert in panel $\mathbf{d}$. The cytoplasm of glandular cells showed a diffuse positive reaction, whereas the nuclei were completely negative. Glandular lumen was filled by a secretion strongly positive for the anti CLU antibody. (f) Low-power field (magnification $\times 10)$ of normal mucosa of the same patient distant from the neoplasia. Colonic glandular cells were faintly positive for the anti-CLU antibody.

protein, as reported earlier. In fact, clusterin determination depends strongly on the protein-protein binding, $\mathrm{pH}$, and temperature (13).

Therefore, the same samples were also analyzed by dot blot. This technique, based on the immobilization of the sample on a poly-vinyl-diene difluoride membrane, was chosen to avoid the hydrophobic regions of clusterin to contact the hydrophobic regions of other proteins (or other molecules of clusterin) and form "clusters" that could precipitate and be washed away. The dot blot analysis (Figure 3b) on human sera from CRC patients (CRC, $n=35$ ) and no cancerous individuals (controls, $n=25$ ) displayed statistically significant differences in the clusterin levels. In fact, clusterin concentration was $82.8 \pm 26.9 \mu \mathrm{g} / \mathrm{ml}$ in CRC cancer patients and $57.8 \pm 19.3 \mu \mathrm{g} / \mathrm{ml}$ in controls (CRC vs. controls: $P=0.0002)$.

\section{Clusterin level in stool increases in cancer patients}

To avoid the interference of the increased level of clusterin in blood owing to other not tumoral or tumoral diseases (cancer of the breast, prostate, testicle, ovary, central nervous system, hemolymphopoietic system), the level of clusterin was determined in the stool of the CRC patients. Clusterin determination was performed both by commercial and by oligoclonal antibodies. A preliminary observation on the integrity of the protein in stool was performed by western blot (Figure 4a). Dot blot analysis of fecal extracts from cancer patients $(n=28)$ compared with controls $(n=25)$, provided significant differences with mean values of $47.5 \pm 19.6$ and $26.8 \pm 12.8 \mu \mathrm{g} / \mathrm{g}$, respectively (CRC vs. controls: $P<0.000$ ) (Figure 4b). The analysis was performed three times in triplicate. Moreover, the analysis in patients $(n=16)$ undergone to 

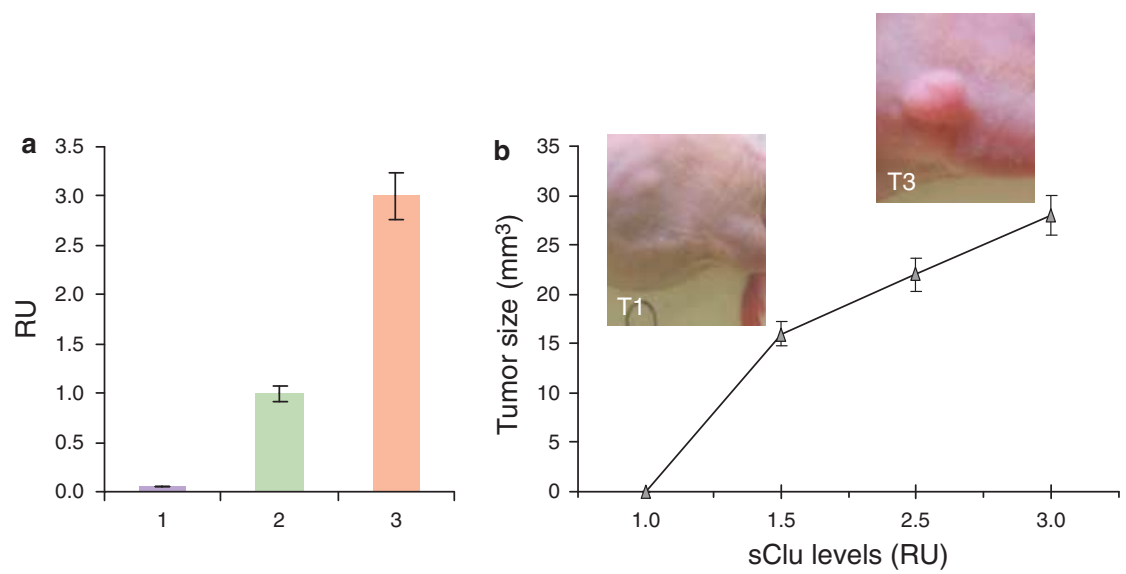

Figure 2. Clusterin level in the culture supernatants of the ex vivo-isolated cells (a) from healthy (2) and neoplastic colonic mucosa (3), evaluated by enzyme-linked immunosorbent assay (ELISA). Complete culture medium alone was used as control (1). Values are means ( \pm s.d.) of three independent experiments. Dot blot analysis of serum clusterin in tumor xenograft model (b). Secreted clusterin levels in Caco-2 cells uninjected nude mice (basal level, 1 ) and in human tumor-bearing mice are reported as relative units (RU) ( $x$ axis) and are plotted vs. the corresponding tumor sizes ( $y$ axis). Values are means ( \pm s.d.) of three independent experiments. T1 and T3: tumor-bearing mice after 15 and 25 days after injection of tumor cells.
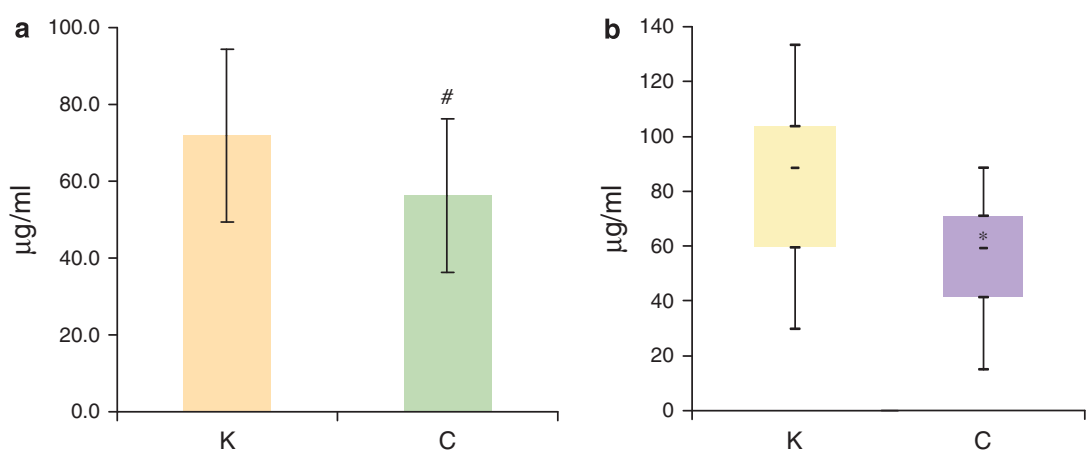

Figure 3. Analysis of clusterin in serum. (a) Clusterin concentration evaluated by enzyme-linked immunosorbent assay (ELISA) in the serum of colorectal cancer (CRC) patients $(K)$ and healthy individuals $(C)$. Values are means ( \pm s.d.) of three independent experiments. \#Indicates no statistical significance by ELISA detection ( $P=0.07$ Student's $t$-test). (b) Clusterin levels in $\mu \mathrm{g} / \mathrm{ml}$ are reported, as obtained by dot blot analysis in sera of colorectal cancer patients $(K=35)$ and healthy individuals $(C=25)$. Box plots show the distribution of data values, as means of three independent experiments. The bar inside the box indicates the median of distribution, the box spanning the interquartile range, and the external bars represent the lowest and highest data points. Asterisk indicates the statistically significant difference ( $\left.{ }^{\star} P=0.0002\right)$.

endoscopic polipeptomy showed no significant differences in sCLU level between low-grade dysplasia adenomas and control samples $(P=0.170)$ and between high-grade adenomas and CRC samples $(P=0.065)$. Conversely, the two groups of dysplastic lesion, i.e., low- and high-grade dysplasia adenomas, showed significant differences in the levels of sCLU $(P=0.003)$. These data confirmed the observations that we have published earlier, where only the high-grade dysplasia adenomas (advanced adenomas) and CRC tissues displayed an overexpression of sCLU isoform (7). Also, a significant correlation between clusterin values in stool and stage of cancer disease was found $(P=0.05)$.

We also constructed ROC curves by plotting sensitivity (the proportion of true-positive results) vs. 1-specificity (the proportion of false-positive results) both in sera and in stool (Figure 5) and determined the corresponding AUC to show the ability of secreted clusterin to discriminate nonneoplastic (controls) from neoplastic lesions (CRC). The AUC is a useful onestatistic summary of the diagnostic accuracy of the assay. Both the tests showed a moderate diagnostic accuracy, AUC being in the interval 0.7-0.9 (16).

The AUCs of ROCs from stool and blood clusterin were analytically compared (17), and nonsignificant differences were found between the two methods $(P=0.686)$, despite the AUC from stool assay being 0.81 (95\% CI 0.695-0.926), whereas in blood it was 0.77 (95\% CI 0.641-0.901). The ROC curves also provided several cutoff points to show the tradeoff between sensitivity and specificity at different cutoff values. For dot blot assay in blood, the optimal threshold was $88.5 \mu \mathrm{g} / \mathrm{ml}$ corresponding to $55.6 \%$ (95\% CI 39.7-69.9) sensitivity and $100 \%$ (95\% CI $85.1-100)$ specificity, whereas the stool test reached $66.7 \%$ (95\% CI 47.8-81.4) sensitivity and $84 \%$ (95\% CI 65.3-93.6) specificity at the selected cutoff value of $34.6 \mu \mathrm{g} / \mathrm{g}$ (Table 3 ). 

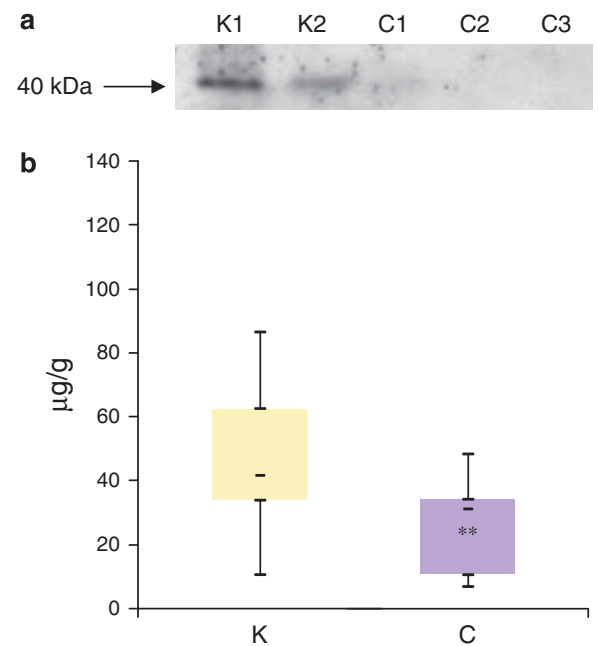

Figure 4. Analysis of clusterin in stool. (a) Detection of clusterin protein extracts from stool of colorectal cancer (CRC) patients $(K 1, K 2)$ and healthy individuals ( $\mathrm{Cl}-\mathrm{C3}$ ) performed by western blot. A $40 \mathrm{kDa}$ band can be detected corresponding to the cytoplasmic/secreted isoform. (b) Clusterin levels in $\mu \mathrm{g} / \mathrm{g}$ are reported as obtained by dot blot analysis in the stools of CRC patients $(K=28)$ and healthy individuals $(C=25)$. Box plots show the distribution of data values, as means of three independent experiments. The bar inside the box indicates the median of distribution with the box spanning the interquartile range. The external bars represent the lowest and highest data points. Asterisks indicate the statistically significant difference $\left({ }^{* *} P<0.000\right)$.

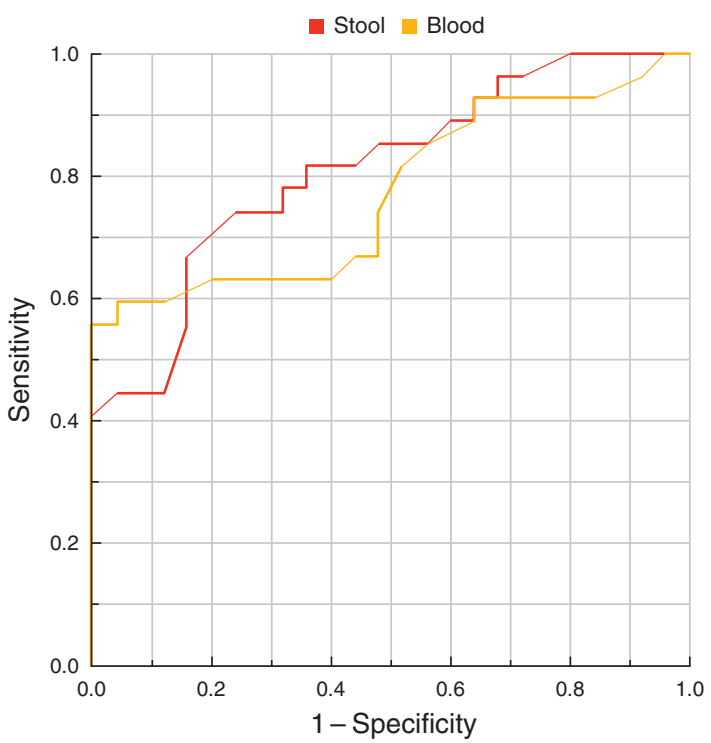

Figure 5. Secreted clusterin isoform discriminates between colorectal cancer disease and nonneoplastic controls. Receiver operating characteristic curves for blood (orange) and stool (dark red) tests are shown. Both the tests show moderate diagnostic accuracy, area under the curve being 0.81 (95\% confidence interval $(\mathrm{Cl}) 0.69-0.92$; $P<0.000$ ) for stool and 0.77 (95\% $\mathrm{Cl} 0.64-0.90 ; P<0.001)$ for blood assay, respectively, (nonparametric assumption, SPSS software).

\section{DISCUSSION}

The early diagnosis and disease recurrence of CRC are central to the effective treatment of this pathology. In addition, molecular markers indicative for the disease relapse or micrometastasis expansion actually are poorly available. In the current report, we showed for the first time that in colon cancer the upregulated cytoplasmic CLU is extracellularly released both in blood and in stool. By developing a sensitive method, we found a significant increase of clusterin both in the blood and stool of cancer-affected patients, highlighting its potential value as a new diagnostic biomarker for a noninvasive test for colon cancer screening.

There is a consensus that CRC screening is effective to prevent this disease in many cases (6). Owing to CRC screening, the incidence of this tumor has dropped in recent years. There is less consensus regarding optimal screening strategies, as sensitivity, specificity, and patient acceptance limit current options. To overcome these barriers, a range of approaches, including proteomics-based testing, stool genetic testing, radiological imaging, and enhanced endoscopies, have been the focus of intense research $(1,3,4,18,19)$. A useful diagnostic assay must be sensitive, must detect cancer at the onset, and must have a high specificity to minimize false positives that necessitate expensive and invasive examination. Stool testing, unlike other conventional screening approaches, is noninvasive and requires no cathartic preparation. However, widely used fecal occult blood test yields frequent false-negative and false-positive results that lower screening effectiveness and raise program costs. According to literature, overall new stool tests for CRC diagnosis have a higher sensitivity, whereas specificity is still to be defined. In particular, specificities of about $95 \%$ have been reported for tests based on the detection of genetic mutations occurring in the tumoral tissues, but not in the early stage, and these are not present in all cases. On the other hand, markers such as calprotectin may represent both a marker of cancer disease and of bowel inflammation, leading to nearly $30 \%$ false-positive results $(20,21)$. Recently, a high-specific serum testing for colon cancerspecific antigen 2 and 4 has been proposed, but the limitation of this test is that not all colon cancers may express the nuclear matrix protein colon cancer-specific antigen 2 and 4 (20-30\%) and therefore a multiple marker testing is needed (22).

We have demonstrated earlier a tumor-related pattern shift of CLUs production in colon tumorigenesis observing that sCLU was upregulated in the cytoplasm of neoplastic cells and correlated with the stage of the disease (8). In fact, clusterin is expressed in the nucleus and weakly expressed in the cytoplasm of normal colonocytes, in particular in the luminal crypt side. Conversely, CLU was found elevated in the cytoplasm of colon carcinomas (50 out of 50 observed) and its production was shown to correlate with the nodal status. The dot blot assay for CLU detection in stool shows diagnostic accuracy with satisfactory values of sensitivity and specificity, compared with the other noninvasive cancer screening tests widely used. Our preliminary observations obtained by stool analysis clearly point out that the increase of CLU in cancer patients is significant not 
Table 3. Receiver operating characteristic (ROC) curve summary statistics

$\begin{array}{lccccccc}\text { Classifier } & \text { AUC } & \text { Inverted } & \text { ACC } & \text { OT } & \text { fp } & \text { tp } & \text { N } \\ \text { Stool } & 0.8104 & + & 0.7500 & 34.6000 & 0.1600 & 0.6667 & 25 \\ \text { Blood } & 0.7711 & + & 0.7692 & 88.5000 & 0.0000 & 0.5556 & 25 \\ \begin{array}{l}\text { ACC, maximal accuracy; } \\ \text { of positive instances; }\end{array} & + \text { area under the ROC curve; fp, false-positive rate (1-specificity) at OT; N, number of negative instances; OT, optimal threshold; P, number }\end{array}$

only compared with healthy individuals, but also compared with patients affected by systemic or bowel inflammatory pathologies and benign lesions of the colon. Preliminary data obtained from the analysis of adenomas with low-grade dysplasia, endoscopically removed and histologically characterized, showed no significant differences in sCLU level in stool compared with that of healthy individuals. From an examination of the stool samples, it is ruled out that in a healthy population clusterin is not normally present in high amounts as a discarding product and could constitute a point-matched control to cross with blood clusterin level. Data obtained by the determination of clusterin in blood by dot blot test showed a $56 \%$ sensitivity calculated by the ROC curve and, considering the entire study population, a $100 \%$ specificity. However, the high specificity based on the blood CLU level could be due to the cases selection that did not include individuals with inflammatory pathologies, diabetes, autoimmune diseases, and cardiocirculatory pathologies reported earlier to present high CLU levels in serum (13).

More clinical cases could be helpful to define the clinical relevance of CLU level in the stool and blood of individuals affected by inflammatory pathologies, with respect to CRC patients. Moreover, a significant increase of sCLU in stool was evidenced not only in CRC patients but also in individuals affected by high-grade adenomas, suggesting that clusterin could represent a useful biomarker to detect cancer at a preventable stage in neoplastic precursor lesions. It is worthy of note that a significant decrease of CLU level was found in a small cohort of follow-up patients $(n=8) 6-8$ months after tumor removal (data not shown), indicating its potential role as a progression-free survival marker.

Overall, these data propose sCLU as a new marker of onset, prognosis, and relapse of colon cancer. Large trials with full clinical data and follow-up available on the sCLU detection in stool will need to confirm its potential value for noninvasive, effective, and low-cost screening programs for CRC detection. Moreover, studies on the molecular mechanisms that regulate the activation of clusterin promoter and its isoforms shifting could provide new molecular targets for specific anti-neoplastic therapies.

\section{CONFLICT OF INTEREST}

Guarantor of the article: Luigi G. Spagnoli, MD. Specific author contributions: Pucci S. contributed to the idea for the study, performed the basic conceptual work, and prepared the paper; L.G. Spagnoli contributed to the idea for the study, performed the histopathological evaluation, and contributed to the paper; E. Bonanno added conceptual ideas, performed the histopathological evaluation, and contributed to the paper; P. Mazzarelli contributed to the paper with fundamental conceptual ideas; F. Sesti performed laboratory work and added to conceptual ideas; A. Mauriello and G. Biondi Zoccai performed statistical analysis; F. Ricci, F. Rulli and G. Galatà enrolled patients and collected samples. Financial support: This work was supported in part by Alleanza Contro il Cancro (ACC)-Istituto Superiore di Sanità (ISS) Art. 3 DM 21 luglio 2006-Programma Straordinario di Ricerca Oncologica 2006. Programma 3 "Rete solidale e collaborazioni internazionali"; Ricerca Oncologica: Progetto Ordinario IRCCS.

Potential competing interests: None.

Ethics approval: All animals were approved by Institutional Animal Care and Use of University of Rome Tor Vergata. The study is in accordance with the Declaration of Helsinki.

\section{Study Highlights}

\section{WHAT IS CURRENT KNOWLEDGE}

Noninvasive colorectal cancer (CRC) screening can reduce CRC mortality by $15-33 \%$.

Fecal occult blood tests yield frequent false-negative and false-positive results that lower screening effectiveness and raise the program costs.

The overexpression of the prosurvival isoform of clusterin (sCLU) correlates with the neoplastic phenotype in colorectal mucosa and with CRC progression.

\section{WHAT IS NEW HERE}

The upregulated sCLU is extracellularly released both in blood and in the stool of CRC patients.

A new sCLU immuno-dosage in blood and stool efficiently discriminates between CRC patients and healthy individuals.

Clusterin detection in stool represents a valuable tool to improve the effectiveness and efficiency of large-scale clinical cancer screening.

\section{REFERENCES}

1. Levin B, Lieberman DA, McFarland B et al. Screening and surveillance for the early detection of colorectal cancer and adenomatous polyps, 2008: a joint guideline from the American Cancer Society, the US Multi-Society Task Force on Colorectal Cancer, and the American College of Radiology. CA Cancer J Clin 2008;58:130-60. 
2. Walsh JME. Colorectal cancer screening. JAMA 2003;289:1288-96.

3. Burch JA, Soares-Weiser K, St John DJB et al. Diagnostic accuracy of faecal occult blood tests used in screening for colorectal cancer: a systematic review. J Med Screen 2007;14:132-7.

4. Winawer S, Fletcher R, Rex D et al. Colorectal cancer screening and surveillance: clinical guidelines and rationale: update based on new evidence. Gastroenterology 2003;124:544-60.

5. Winawer SJ. The multidisciplinary management of gastrointestinal cancer. Colorectal cancer screening. Best Pract Res Clin Gastroenterol 2007;21:1031-48.

6. Ouyang DL, Chen JJ, Getzenberg RH et al. Noninvasive testing for colorectal cancer: a review. Am J Gastroenterol 2005;100:1393-403.

7. Pucci S, Bonanno E, Pichiorri F et al. Modulation of different clusterin isoforms in human colon tumorigenesis. Oncogene 2004;23:2298-304.

8. Redondo M, Villar E, Torres-Muñoz J et al. Overexpression of clusterin in human breast carcinoma. Am J Pathol 2000;157:393-9.

9. Leskov KS, Klokov DY, Li J et al. Synthesis and functional analyses of nuclear clusterin, a cell death protein. J Biol Chem 2003;278:11590-600.

10. Yang CR, Leskov K, Hosley-Eberlein K et al. Nuclear clusterin/XIP8, an $\mathrm{X}$-ray-induced Ku70-binding protein that signals cell death. Proc Natl Acad Sci USA 2000;97:5907-12.

11. Pucci S, Bonanno E, Pichiorri F et al. The expression and the nuclear activity of the caretaker gene ku86 are modulated by somatostatin. Eur J Histochem 2004;48:103-10.

12. Pucci S, Mazzarelli P, Sesti F et al. Interleukin-6 affects cell death escaping mechanisms acting on Bax-Ku70-Clusterin interactions in human colon cancer progression. Cell Cycle 2009;8:1-9.
13. Trougakos IP, Poulakou M, Stathatos M et al. Serum levels of the senescence biomarker clusterin/apolipoprotein J increase significantly in diabetes type II and during development of coronary heart disease or at myocardial infarction. Exp Gerontol 2002;37:1175-87.

14. Morrissey C, Lakins J, Moquin A et al. An antigen capture assay for the measurement of serum clusterin concentrations. J Biochem Biophys Methods 2001;48:13-21.

15. Stejskal D, Fiala RR. Evaluation of serum and urine clusterin as a potential tumor marker for urinary bladder cancer. Neoplasma 2006;53:343-6.

16. Akobeng AK. Understanding diagnostic tests 3: receiver operating characteristic curves. Acta Pediatrica 2007;96:644-7.

17. Vergara IA, Norambuena T, Ferrada E et al. StAR: a simple tool for the statistical comparison of ROC curves. BMC Bioinformatics 2008;9:265.

18. Jankowski JA, Odze RD. Biomarkers in gastroenterology: between hope and hype comes histopathology. Am J Gastroenterol 2009;104:1093-6.

19. Bonanno E, Rulli F, Galatà G et al. Stool test for colorectal cancer screening: what is going on? Surg Oncol 2007;16:S43-5.

20. Tibble J, Sigthorsson G, Foster R et al. Faecal calprotectin and faecal occult blood tests in the diagnosis of colorectal carcinoma and adenoma. Gut 2001;49:402-8.

21. Lemn ES, Shoen RE, Magheli A et al. Evaluation of cancer-specific antigen 2 as a potential serum marker for colorectal cancer. Clin Cancer Res 2008;14:1349-54.

22. Giuttet L, Bouvier V, Vallee JP et al. Comparison of guaiac based and an immunochemical faecal occult blood test in screening for colorectal cancer in a general average risk population. Gut 2007;56:210-21. 